\title{
基于古代绘画的古诗自动生成方法
}

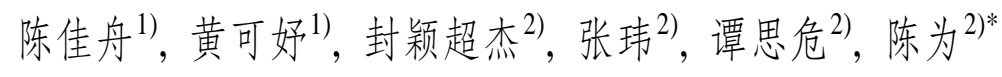 \\ ${ }^{1)}$ (浙江工业大学计算机科学与技术学院 杭州 310012) \\ 2) (浙江大学 CAD\&CG 国家重点实验室 杭州 310058) \\ (chenwei@cad.zju.edu.cn)
}

\begin{abstract}
摘 要: 中国题画诗是世界艺术史上一种极为特殊的艺术形式, 它结合了中国古代文学和美术, 诗与画相得益彰、浑 然一体. 为实现计算机为画题诗的功能, 提取和使用古代绘画的多句现代文描述, 提高古诗对绘画的文学表达能力, 提出一种基于古代绘画的古诗自动生成方法. 首先根据古画建立多句现代文标注数据集, 然后通过改进的图像描述 方法提取古画语义特征得到多句现代文描述, 最后通过双向 LSTM 编解码框架将这些现代文描述转化为律诗. 针对 宋代小品画的实验表明，所提方法生成的古诗前后连贯、押韵规律，符合古画原有的内容且符合上下文语境; 用户调 查评估结果表明了该方法的有效性，其内容一致性和总体满意度等指标均优于基于关键词的方法.
\end{abstract}

关键词: 古代绘画; 古诗; 题画诗; 图像描述

中图法分类号: TP391.41 DOI: 10.3724/SP.J.1089.2021.18633

\section{Automatic Poetry Generation Based on Ancient Chinese Paintings}

\author{
Chen Jiazhou $^{1)}$, Huang Keyu ${ }^{1)}$, Feng Yingchaojie ${ }^{2)}$, Zhang $\mathrm{Wei}^{2)}$, Tan Siwei ${ }^{2)}$, and Chen $\mathrm{Wei}^{2{ }^{*}}$ \\ ${ }^{1)}$ (College of Computer Science and Technology, Zhejiang University of Technology, Hangzhou 310012) \\ ${ }^{2)}$ (State Key Laboratory of CAD\&CG, Zhejiang University, Hangzhou 310058)
}

\begin{abstract}
The Chinese painting poem is a very special art form in the history of world art. It combines ancient Chinese literature and fine arts, complements each other and blends together. In order to obtain computer-based painting poetry, an automatic poetry generation is proposed based on ancient Chinese paintings. It extracts multiple sentences from ancient paintings, which improves the literary expression ability of ancient poems in paintings. Firstly, a multi-sentence annotation data set for ancient paintings is established, and then semantic features of ancient paintings are extracted through an improved image captioning method. Finally, these modern text descriptions are converted into a four-character poem through a two-way LSTM encoding and decoding framework. The experiment on the paintings of the Song Dynasty demonstrates that the coherent and prosodic poems generated by our method are consistent with the original content and context of the ancient paintings. User study shows that the content consistency and user satisfaction of our method are better than keyword-based methods, which proves the validity of the proposed method
\end{abstract}

Key words: ancient paintings; ancient poems; inscription poems; image caption

收稿日期：2020-08-29; 修回日期：2020-11-13．基金项目：浙江省文物科技保护项目(2020014). 陈佳舟(1984一), 男，博士，副 教授, 硕士生导师, CCF 会员, 主要研究方向为计算机图形学、数字人文; 黄可妤(1998一), 女, 硕士研究生, 主要研究方向为计算机 图形学、人工智能; 封颖超杰(1997一), 男, 硕士研究生, 主要研究方向为数字人文; 张玮(1988一), 女, 硕士, 工程师, 主要研究方向 为可视化; 谭思危(1997一), 男, 硕士研究生, 主要研究方向为数据科学; 陈为 (1971一), 男, 博士, 教授, 博士生导师, CCF 会员, 论 文通讯作者, 主要研究方向为可视化与可视分析. 
中国古代绘画一般是在绢纸上用毛笔耎取水、 墨、颜料等绘制而成. 大多数古画绘制山水、亭台 楼阁、人物及花鸟兽鱼等, 是我国一种极具特色的 绘画方式. 中国的题画诗是世界艺术史上一种极 其特殊的美学现象. 中国画起源于古代，文字与画 在当初是没有歧异的，也有书画同源的说法. 画是 诗的载体, 诗是画的延伸, 诗和画相辅相成, 使文 学和美术浑然一体.

近些年来，计算机作诗不断发展，为了能够让 计算机自动作出与古诗相媲美的诗歌, 出现了各 种方法以实现机器作诗的效果. 如果能够使用机 器学习的方法, 根据绘画内容自动生成相应的古 诗, 实现 “为画题诗”的功能, 通过诗传达画外之 意, 不仅能开阔视野, 而且能丰富画面的意念和启 发观赏古画时的想象.

在作诗方面，现有的古诗生成方法主要以文 字输人为主, 包括基于关键词和单句话的方法. 但 前者在描述动作和方位等信息上有所欠缺, 后者 内容描述不够丰富, 难以形成完整的诗.

本文的主要思路是先为古画生成现代语义描 述, 再通过语义描述生成古诗, 提出了一种基于多 句现代语义描述的为画题诗方法. 该方法避免了 从古画到古诗的直接生成, 解决了上述基于关键 词和单句话的方法在内容提取上的不足.

通过建立古画多句现代文标注数据集，能够 弥补现有图像描述方法数据集局限于自然图像的 不足. 本文提出一种基于图像描述的多句现代文 语义描述的生成方法, 输出的多句语义描述包含 动作和方位等信息，内容更加全面且贴合于古画; 以及一种基于编码器-解码器模型 ${ }^{[1]}$ 的多句现代文 自动生成古诗的方法，不仅充分利用了现代文内 容, 而且保持了古诗的韵律和结构. 支持多句白话 文输人，且能在此基础上自行发挥.

\section{1 相关工作}

\section{1 图像描述}

图像描述是指以图像为输人, 通过模型和计 算输出对应图像的自然语言描述. 该领域是计算 机视觉和自然语言的结合. 近几年图像描述领域 开始快速发展, 早期的方法是以传统的机器学习 作为基础 ${ }^{[2]}$, 包括使用图像处理的算子提取出图像 的特征，使用支持向量机等进行分类，以得到图像 中的目标，再将得到的目标及其属性作为生成句 子依据. 具有代表性的是 Kulkarni 等 ${ }^{[3]}$ 和 Farhadi 等 ${ }^{[4]}$ 较早期的工作. 在 Vinyals 等 ${ }^{[5]}$ 的方法出现之
前, 编码器-解码器结构 ${ }^{[6]}$ 是在机器翻译上使用的. 受这种编码器-解码器的结构启发, Vinyals 等 ${ }^{[7]}$ 和 Karpathy 等 $^{[8]}$ 几乎同期提出套用机器翻译中的这 套结构, 把编码源文本文字(编码器部分)的循环 神经网络(recurrent neural networks, RNN)替换成 图像编码，前者使用卷积神经网络 (convolution neural networks, CNN) ${ }^{[9]}$ 对图像进行识别, 后者使 用 VGGNet(visual geometry group net) ${ }^{[10]}$, 将对图 像进行编码获得的图像特征向量传人至解码器. 在解码器长短期记忆网络(long short-term memory, LSTM) ${ }^{[11]}$ 处出现 gLSTM(group long short-term memory) ${ }^{[12]}$, 以实现图像传人 LSTM 及 att-LSTM (attention long short-term memory $)^{[13]}$, 进而解决传人至全 局特征信息的问题, 再通过解码器把图像特征向 量转换成一句序列. 从机器翻译的角度来看, 此处 源文字就是图像，目标文字就是生成的描述.

看图说话的算法框架在国内外已有了一定的 研究成果, 但大多数都是基于开源的数据集, 如 MSCOCO2014, Flickr30k 和 Flickr8k 等 ${ }^{[14]}$. 这些数 据集由照片组成, 即真实人景物组成的画面. 而中 国古代绘画追求神似, 注重意境, 其以墨线作为造 型的主要手段, 且物体之间的依赖程度较高, 需要 依靠其他物体判断河流等以及人物的行为动作. 因此, 现有的图像描述公开数据集无法满足用于 识别中国特色的古画. 看图说话的算法输出为单 句序列，但在图像的内容上单句描述提供的信息 较少, 难以满足整首诗的生成.

与通过 Faster R-CNN(region convolution neural networks ${ }^{[15]}$, Mask R-CNN ${ }^{[16]}$ 和 Libra R-CNN ${ }^{[17]}$ 等 目标检测方法使用关键词作为古画的描述相比, 现代语句不仅能够描述出古画中包含的景物和人, 也能描述它们的其他属性(如人的动作、神态)和互 相关系(如位置关系). 如果生成的诗句在这些方面 和古画不相符，那么画和诗之间的关联性将大打 折扣.

\section{2 古诗自动生成}

古诗词是中华文学宝库中的瑰宝, 是语言的 精华, 是智慧的结晶. 古今中外的诗人写下了无数 美丽的诗篇, 经过时间的磨炼, 这些诗歌已经成为 超越时空的不朽经典. 古诗自动生成算法一直是 自然语言处理领域中一个重要的研究方向, 已有 的研究成果, 其中最主要的形式是根据一个或数 个关键词生成一首诗. 周昌乐等 ${ }^{[18]}$ 将遗传算法用 于宋词的生成，从词语库中选择与关键词相关程 度高的词语构成候选词语空间, 使用遗传算法迭 代生成优秀的子代词句. He 等 ${ }^{[19]}$ 将统计机器翻译 
方法应用于诗词的生成，根据关键词生成第 1 句诗 句后, 采用机器翻译的方式生成后续诗句. Zhang 等 ${ }^{[20]}$ 也是根据关键词生成符合韵律的首句候选项， 使用 RNN 选择候选句并生成后续诗句. $\mathrm{Yan}^{[21]}$ 提出 从关键词中捕获全局信息表示, 用于指导每行诗 句的生成，同时还提出了一种“迭代抛光”的模式， 模拟人类对诗句不断改进的过程. Yi 等 ${ }^{[22]}$ 提出了 一种带有工作记忆的写诗模型, 将关键词作为主 题记忆保存在工作记忆中, 每次以注意力的形式 从读取工作记忆用于生成诗句.

在机器看图写诗方面, 邢硕 ${ }^{[23]}$ 提出使用关键 词作为中间媒介实现该功能. 但提取高层语义特 征词的方法对于古画这种强调写意的图像识别能 力较差, 导致生成的内容不够完整, 在后续的作诗 上存在问题.

另外一种诗词的生成形式是根据一句或多句 的现代语言描述, 这种形式相比于关键词会保留 更多相关信息在诗句中, 使生成的古诗能够更加 与古画贴合. 不同的学者处理现代语言描述的方 式也有所不同. Wang 等 ${ }^{[24]}$ 提出了一种基于规划的 神经网络, 对输人的语言序列提取关键词, 并对关 键词列表进行扩充使其符合数量要求, 然后由这 些关键词生成相应的诗句. 王东等 ${ }^{[25]}$ 提出了一种 现代文到古诗的转换方法, 通过神经网络直接对 现代文进行语义理解, 并且根据平入和韵律等规 则输出语义相符的诗句. 在现代文到古诗的转换
的过程中使用注意力机制加强对现代文中重要内 容的关注程度. 该方法使现代文语句中的逻辑关 系得以保留并在诗中体现, 因此可以保留图像中 的更多信息，使生成的诗句和图像具有更高的一 致性. 但图像的现代文描述语句通常少于 4 句, 部 分诗句的生成过程会缺少现代文的输人, 这对神 经网络提出了更高的要求. 因此, 本文提出了一种 改进的现代文到诗的神经网络模型.

\section{2 本文方法整体框架}

本文将基于计算机的为画题诗拆分为 2 部分, 首先根据古画生成多句现代文, 再根据多句现代 文生成古诗，如图 1 所示.

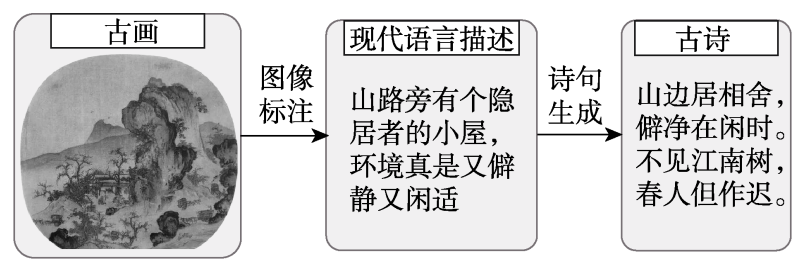

图 1 本文主要思路

与直接由画作诗相比, 本文使用多句现代文 能够以一对一的方式生成绘景诗句, 这样能在景 物方面增添更多细节, 描写也更加全面, 且为后续 提供更多具有意象的物以抒发相应的情感. 具体 框架如图 2 所示.

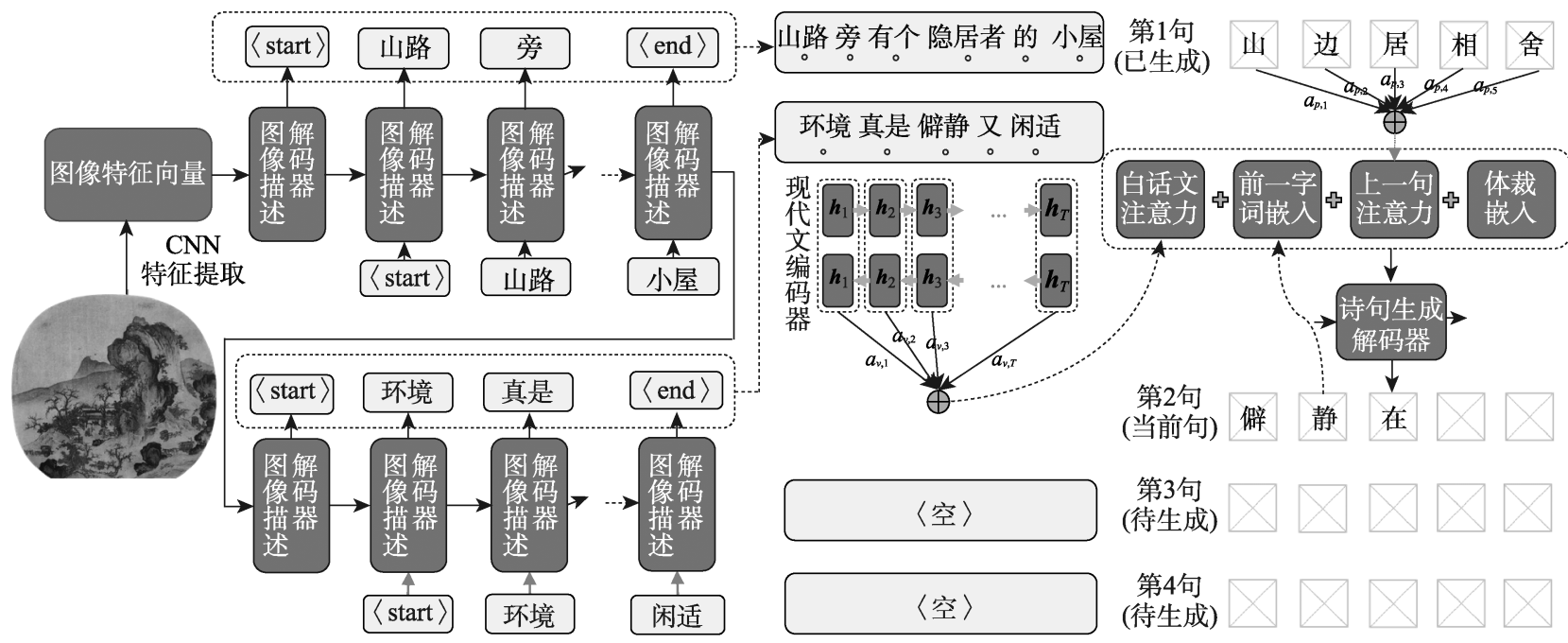

图 2 本文方法整体框架

本文通过宋代小品画建立多句现代文描述标 注数据集，基于图像描述方法实现了古画的“看图 说话”, 该方法使用编码器-解码器框架, 将 CNN 和 LSTM 结合, 构造一个多句图像描述生成的深
度学习网络模型, 能快速地对古画进行识别, 并生 成多句带有形容词的现代文描述.

在作诗方面, 为了实现基于多句现代文的古 诗生成, 本文提出了一个基于编码器-解码器模型 
的古诗生成模型, 以多句现代文作为输人, 并结合 平入和押韵等诗词格律约束, 生成连贯的诗句. 同 时, 应用了注意力机制使模型聚焦于重要的、相关 性高的现代文片段.

值得指出的是，通常一首诗的 4 句不全为写实 诗句, 部分写意的诗句生成就没有现代文信息的 输人, 这就要求生成模型能够根据诗句的上下文 语境生成合适的诗句. 因此, 模型还需要将注意力 聚焦于上一句诗的字词中，对上一句诗的注意力 计算结果不仅有助于提高诗句的内容一致性，也 使上下 2 句诗在句式上可能产生对仗的效果.

\section{3 本文方法实现}

\section{1 多句语义描述数据集}

多句语义描述数据集主要分为图片集和标注 集. 本文主要使用宋代小品画作为图片集. 其特点 为画面篇幅较小, 却能够将完善的内容和细淢的 笔墨技巧展现出来. 小品画中包含了许多不同的 题材, 展现了不同的意境, 也能给观看者无限的联 想, 为其题上一首诗更是能相辅相成, 锦上添花.

标注集中包括图片信息以及为每张图片进行 人为标注的 5 个多句描述语句, 一共包含 1000 条 描述语句. 标注的依据来自画作的解析和背景. 根 据画作情感为标注集添加带有情绪色彩的词汇, 不仅能为后续作诗提供帮助, 还能从情感上对不 同画作有所区分.

由于古画中包含如江河等这种需要通过周围 的物体来展现的内容, 以及需要通过与环境的交 互分析产生的人物动作, 标注的语句需要尽量体 现物体之间的联系. 描述语句标注的规则为: 首先 说明主要人、物和动作，充分展现物体之间的联系; 再根据画的解析和背景增加合适的修饰词汇以丰 富语句. 多句话的标注集既能更加全面地描绘画 面, 又能有主次地说明内容为作诗服务.

\section{2 图像描述的深度学习网络模型}

Show and Tell 模型是通过神经网络和概率框 架生成图像描述. 在图像描述任务中, 输人的是图 像, 输出的是单词序列. 本文使用 InceptionV3 $3^{[26]}$ 提取图片的特征, 参数量远小于 VGGNet, 因此可 以具备系统的训练速度和针对性分类准确度.

模型解码器端利用递归神经网络, 把可变长 度的输人编码作为一种固定长度的特征向量, 并 将其输人至解码端获得输出的结果序列. 给定图 像正确描述概率为

$$
\theta^{*}=\arg \max \sum_{(I, V)} \lg p(V \mid I ; \theta)
$$

其中， $I$ 表示图像; $V=\left\{v_{1}, v_{2}, \cdots, v_{n}\right\}$ 表示图像的描 述序列集, $\theta$ 表示模型的参数. $n$ 表示描述语句的 数量, 本文的数据集采用 $n=2$, 但本文方法可以 方便地扩展到 $n \leqslant 4$ 的其他情况. 对于律诗, 本文 允许 $n<4$ 的优点不仅仅在于数据集的灵活性, 还 在于作诗的非写实部分的发挥. 进一步的讨论会 在本文自动作诗部分展开.

$v_{1}$ 是一个句子，且长度没有限制； $w_{t}$ 是句子 中的词汇, 使用链式求导模拟 $w_{0} \sim w_{N}$ 上的联合概 率, 计算对数概率和为

$$
\lg p(w \mid I)=\sum_{t=0}^{N} \lg p\left(w_{t} \mid I, w_{0}, \cdots, w_{t-1}\right)
$$

其中， $N$ 表示特定示例的长度. 通过随机梯度下降, 对式(2)中的对数概率和进行优化. 本文使用 LSTM 作为解码器, 每个序列中的每个词汇的概率为

$$
p\left(w_{t} \mid w_{0: N-1}, I\right)=\operatorname{LSTM}\left(\boldsymbol{x}_{t}\right), t \in\{0, \cdots, N-1\}
$$
其中,

$$
\begin{gathered}
\boldsymbol{x}_{-1}=\operatorname{CNN}(I) \\
\boldsymbol{x}_{t}=\boldsymbol{e}\left(w_{t}\right), t \in\{0, \cdots, N-1\}
\end{gathered}
$$

$\boldsymbol{e}\left(w_{t}\right)$ 表示 $w_{t}$ 的词嵌人向量.

\begin{tabular}{|c|c|}
\hline 原图 & 输出结果(概率从高到低) \\
\hline & 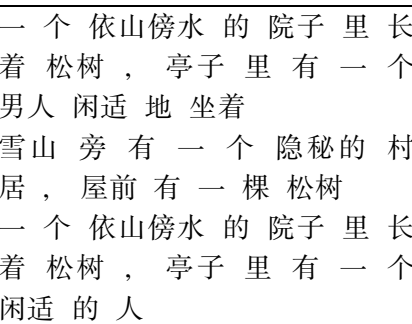 \\
\hline
\end{tabular}

根据图像特征向量或词嵌人向量, 可以预测 到句子中的每一个单词. 表 1 展示了本文方法中对 于古画进行图像描述后生成的中文序列.

表 1 古画图像描述生成结果

为了使描述更加全面，基于上述模型，本文建 立了一个古画标注数据集, 使用多句现代文描述 语句为每一张图片标注, 并对现代文语句进行分 词处理. 数据集中使用的词语具有特征性, 使用词 向量构建单词表能够让输出的词汇更加完整且带 有情感色彩. 本文将序列用 2 句话描述，在第 1 句 话的结束符后紧跟下一句话的开始符, 继续进行 LSTM 操作, 直至第 2 句话输出结束符. 这样的结 构能够保证两句现代文描述语句的生成.

由于古画偏向写意的特点, 通常会以环境中 的其他物体判断具体内容以及人物动作. 如图 $3 \mathrm{a}$ 
所示, 难以通过目标检测等方法识别出江这一重要 的目标物体. 至于人物的行为动作, 人物占据篇幅 较小, 动作也绘制地较为抽象. 如图 3b 所示, 需要 通过远处的山脉和人物脚下的山路来判断人物在山 间远眺, 所以物体之间的联系是至关重要的. 即使 本文中使用 2 句话描述, 但因 LSTM 能够形成记忆, 所以 2 句话之间也会有所联系. 这样不仅能够通过 小舟、岸边等能具体识别的物体, 以输出与江河有 关的词汇, 还能增加后续作诗的上下语句关联程 度. 通过本文含大量修饰词的数据集训练后, 合适 的修饰词会在序列中出现, 丰富了输出的内容.

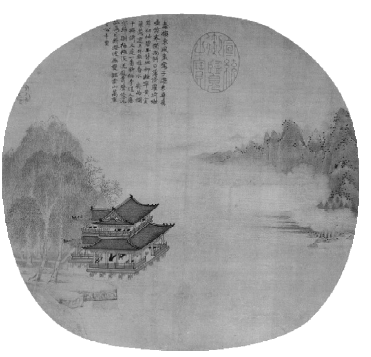

a. 物体识别困难

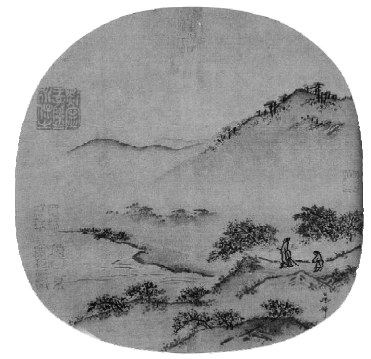

b. 动作检测困难
图 3 古画示意图

\section{3 多句现代文的编码}

图像描述模型根据宋代小品画生成 $n$ 句现代 语言描述 $V=\left\{v_{1}, v_{2}, \cdots, v_{n}\right\}$, 如果 $n<4$, 则部分诗 句会缺失相应的现代文输人, 将这部分缺失的现 代文序列记为 $V_{\text {null }}=\left\{v_{(n+1)}, \cdots, v_{4}\right\}$, 全部现代文序 列 $V_{\text {all }}=\left\{v_{1}, \cdots, v_{4}\right\} . V$ 中的每句现代文描述了古画 的整体或局部内容. 本文使用一个双向 LSTM 对 语句序列 $V_{\text {all }}$ 进行编码, 由于多句描述语句之间存 在着语义的连贯性，因此本文将多句描述语句连 接成一整句现代文语句, 输人到编码器中. 具体步 骤如下.

输人. 现代文 $V_{i}$.

输出. 编码结果 $E_{c}$.

Step1. 使用填充符将每句现代文 $v_{i} \in V_{\text {all }}$ 填充至特 定长度 $L_{v}$.

Step2. 将填充后的现代文连接成一个长序列 $v_{c}=v_{1}+\cdots+v_{4}$, 用字来表示即为 $v_{c}=\left\{w_{j}\right\}_{j=1}^{L_{x} \times 4}, w_{j}$ 表示 长序列中的字符.

Step3. 使用双向 LSTM 对长序列进行编码得到 $E_{c}=\left\{h_{j}\right\}_{j=1}^{L_{L} \times 4}, h_{j}$ 表示 $w_{j}$ 的编码结果.

\section{4 解码器生成古诗}

本文使用 LSTM 作为解码器, 记解码器生成 的诗句 $P=\left\{p_{1}, \cdots, p_{4}\right\}$. 其中, $p_{i}=\left\{y_{t}\right\}_{t=1}^{L_{p}}, y_{t}$ 表示
诗句中的字; $L_{p}$ 表示每句诗的长度;

$$
s_{t}=\operatorname{LSTM}\left(s_{t-1},\left[\boldsymbol{e}\left(y_{t-1}\right) ; c_{t} ; \boldsymbol{g}_{t}\right]\right)
$$

表示 LSTM 的隐状态; $\boldsymbol{e}\left(y_{t}\right)$ 表示 $y_{t}$ 的词嵌人向 量; $\boldsymbol{g}_{t}$ 表示体裁嵌人向量 ${ }^{[22]}$, 则诗句 $p_{i}$ 中的每个 字的概率分布为

$$
p\left(y_{t} \mid y_{1: t-1}, p_{1: i-1}, v_{1: s_{v}}\right)=\operatorname{Softmax}\left(W \times\left[s_{t} ; c_{t}\right]\right)
$$

其中， $c_{t}$ 表示注意力机制的计算结果; $W$ 表示投 影参数. $c_{t}$ 包含了 2 部分的注意力, 一部分来自现 代文, 以下简称现代文注意力; 另一部分来自前一 句诗 $p_{(i-1)}$, 以下简称上一句注意力. 注意力 $c$ 的计 算公式 $A_{t}(S, \boldsymbol{q})$ 为

$$
A_{t}(S, \boldsymbol{q})=\sum_{k=1}^{L} S[k] \times \operatorname{Softmax}\left(\boldsymbol{b}^{\mathrm{T}} \sigma(S[k], \boldsymbol{q})\right)
$$

其中， $\sigma$ 表示非线性层，本文使用 $\tanh$ 函数， $S$ 表 示求解注意力的序列; $\boldsymbol{q}$ 为查询向量; $\boldsymbol{b}^{\mathrm{T}}$ 为线性 投影参数, $k$ 为序列 $S$ 中的位置序号.

现代文注意力指示了诗句的主要内容. 为了强 化现代文 $v_{i}$ 和诗句 $p_{i}$ 在转变过程中的对应关系, 本 文将第 3.3 节中的编码结果 $E_{c}$ 按特定长度 $L_{v}$ 重新 分为 4 句, 即 $E_{c}=\left\{e_{i}\right\}_{i=1}^{4}$. 其中, $e_{t}=\left\{h_{t}\right\}_{t=(i-1) \times L_{v}+1}^{i \times}$. 在诗句 $p_{i}$ 的生成过程中, 将现代文注意力约束在 $e_{i}$ 序列内, 不会被其他诗句的现代文干扰. 现代文 注意力为

$$
c_{v}=A_{t}\left(e_{i}, s_{t}\right)
$$

上一句注意力加强了诗句之间在句式或内容 上相对或相反的联系. 当把注意力放在上一句的 字词上时, 往往更关注字词的含义和词性, 而不是 它们之间的连贯性. 所以，本文将上一句的字词的 词嵌人向量序列作为求解注意力的序列, 即

$$
\begin{array}{r}
e p_{i}=\left\{\boldsymbol{e}\left(y_{t}\right)\right\}_{t=1}^{L_{p}}, \text { 上一句注意力为 } \\
c_{p}=A_{t}\left(e p_{i}, s_{t}\right)
\end{array}
$$

体裁嵌人向量 $\boldsymbol{g}_{t}$ 指示了生成的字符需要遵守 的韵律、句式长度方面的约束. 其中的韵律嵌人指 示了输出字符的平入或韵部, 而长度嵌人指示了 当前诗句的剩余字符生成数量.

\section{4 实验结果与分析}

本文通过使用多句现代文作为媒介，实现从古 画到多句现代文, 再从多句现代文到古诗, 从而达 到为画作诗的效果. 表 2 所示为本文方法生成的结 果. 生成的结果注重平入韵律, 流畅且连贯, 在内 
表 2 本文实验结果

\begin{tabular}{|c|c|c|}
\hline 输人古画 & 输出形式 & 输出结果 \\
\hline & 多句现代文 & $\begin{array}{l}\text { 闲庭中有个人在观潮, } \\
\text { 潮水拍打着江岸. }\end{array}$ \\
\hline & 古诗 & $\begin{array}{l}\text { 闲来有客见前阳, } \\
\text { 水落江头锦谷湘. } \\
\text { 万里之归今日醉, } \\
\text { 愁明不得一杯霜. }\end{array}$ \\
\hline
\end{tabular}

容上充分展现画中的事物，能够表达画中的情感、 意境, 做到诗情画意, 融为一体, 诗与画相辅相成.

将本文方法与其他为画题诗的方法进行比较. 现有的为画题诗方法主要以语义关键词作为画和 诗之间的信息载体，使用单句话的方法本质上也 是通过从句子中提取关键词实现的，其涵盖的信 息量有时还不如关键词丰富，且使用的数据集主 要以现代风格的图片为主, 与本文的应用场景有 较大的差距. 因此, 本文使用关键词提取算法对本 文的古画描述结果提取关键词，使用一种基于关 键词的古诗词生成方法 ${ }^{[22]}$ 生成诗句, 与本文的结 果进行比较.

在实验数据上，本文从古画数据集中随机选 择 30 幅作为测试样本, 将上述 2 种方法生成的结 果进行比较. 本文采用人工评估的方法对结果进 行评分, 评分的指标包括连贯性、内容一致性、意 境相似性和总体满意度. 其中, 内容一致性用来度 量画和诗描写内容的匹配程度, 意境相似性用来 度量画和诗的意境相似程度, 这两者可以较好地 反映画和诗的匹配度. 评分采用 5 分制, 定向邀请 人文学院和艺术学院的 10 位文学研究者以及随机 邀请计算机学院的 20 位普通用户，在未知方法细 节、顺序打乱的情况下对结果进行评分，将评分的 结果求平均值, 得到了如下的评价结果.

从表 3 中的评分可以看出, 无论是文学研究者 还是普通用户，对本文方法的评价都高于关键词 方法. 通过 $\mathrm{T}$ 检验(T-test)结果分析可得，不管是文 学研究者还是普通用户, 本文方法与关键词方法 的评估结果都存在极显著性差异. 其中, 文学研究

表 32 种方法的评价结果

\begin{tabular}{|c|c|c|c|c|c|c|}
\hline 用户 & 方法 & 连贯性 & $\begin{array}{c}\text { 内容 } \\
\text { 一致性 }\end{array}$ & $\begin{array}{c}\text { 意境 } \\
\text { 相似性 }\end{array}$ & $\begin{array}{c}\text { 总体 } \\
\text { 满意度 }\end{array}$ & T-test \\
\hline \multirow{2}{*}{ 文学研究 } & 本文 & 3.871 & 3.752 & 3.740 & 3.813 & \multirow{2}{*}{0.003} \\
\hline & 关键词[22] & 3.674 & 3.533 & 3.573 & 3.595 & \\
\hline \multirow{2}{*}{ 普通 } & 本文 & 3.938 & 3.862 & 3.935 & 3.910 & \multirow{2}{*}{0.001} \\
\hline & 关键词[22] & 3.774 & 3.576 & 3.647 & 3.647 & \\
\hline
\end{tabular}

者的评估差异性略小于普通用户. 在各项指标上, 本文方法都获得了更高的评分, 可见本文方法呈 现的效果更好，说明基于多句现代文的方法能更 好地实现从古画到古诗的生成.

表 4 所示为 2 个测试样本的过程对比, 可以看 到本文方法得到的结果中, 前 2 句诗句和标注信息 有着比较强的对应关系, 也能较好地表达出标注 信息的内容. 而关键词方法因为受到关键词数量 的限制, 导致标注信息的部分内容被舍弃. 表 4 中 关键词方法生成的诗句“旅魂南北渡江河”和关键 词“小船”有着较强的联系, 但由于该方法无法获 取小船和 “停靠”的主谓关系，导致该句诗句和古 画的内容产生了较大的偏差, 而本文方法的结果 与画的内容的一致性较好. 表 4 中关键词方法生成 的诗句“江湖晚放舟”和关键词“环绕” 存在关联, 但 画中没有舟船有关的场景和内容，使诗和画的匹 配程度受到了较大的影响. 本文方法生成的诗句 中前 2 句与画的匹配度较好, 第 3 句中的“雨”没有 在画中出现，但对于画中的场景是可以接受的.

表 4 本文方法与基于关键词方法的古诗生成效果比较

\begin{tabular}{|c|c|c|c|}
\hline 输人古画 & 方法 & 中间产物 & 生成结果 \\
\hline & 本文 & $\begin{array}{l}\text { 溪边停靠着一 } \\
\text { 艘小船; 亭子 } \\
\text { 里有两个人在 } \\
\text { 聊天. }\end{array}$ & $\begin{array}{l}\text { 溪流一岸泛舟船, } \\
\text { 客乐红亭正当来. } \\
\text { 不用高歌如有在, } \\
\text { 今朝应为此山台. }\end{array}$ \\
\hline & 关键词 ${ }^{[22]}$ & $\begin{array}{l}\text { 小船 停靠 } \\
\text { 亭子 }\end{array}$ & $\begin{array}{l}\text { 小屋清风吹客鹅, } \\
\text { 旅魂南北渡江河. } \\
\text { 不知是处湖山好, } \\
\text { 肯与东溪受旧多. }\end{array}$ \\
\hline & 本文 & $\begin{array}{l}\text { 江边有一个隐 } \\
\text { 秘的村居; 山 } \\
\text { 峰高䇯人云, } \\
\text { 云雾环绕. }\end{array}$ & $\begin{array}{l}\text { 江山村远地, } \\
\text { 行断一云峰. } \\
\text { 水落寒沙雨, } \\
\text { 何时两三风. }\end{array}$ \\
\hline & 关键词 ${ }^{[22]}$ & $\begin{array}{l}\text { 山峰 云雾 村 } \\
\text { 居 环绕 }\end{array}$ & $\begin{array}{l}\text { 山翠晓云收, } \\
\text { 江湖晚放舟. } \\
\text { 不堪供寂寞, } \\
\text { 谁识此时留. }\end{array}$ \\
\hline
\end{tabular}

\section{5 结 语}

本文在图像描述和现代文作诗的基础上，提 出了对于图像输出多句现代文语义描述的方法和 通过多句现代文生成古诗的方法, 利用多句现代 文作为中间媒介，将两者相结合，能够实现题画诗 的效果. 整体的思路是将题画诗这一项从画直接 到诗的工作拆分为从画到白话文, 再从白话文到 诗的 2 步工作. 首先对于古画建立相关的多句现代 文标注数据集; 接着通过改进的图像描述方法提 
取古画信息得到多句现代文描述语句; 最后利用 多句描述语句生成古诗. 实验表明，本文方法生成 的古诗前后连贯，内容上丰富有情感，贴合古画且 能充分展现古画内容.

本文方法依赖于标注数据集中的古画内容, 对于出现频率低的内容识别效果较差. 因此, 将来 的工作之一是研究如何准确获得难以识别的内容. 此外，对于题画诗情感方面，可以考虑从画中提取 情感和加人用户情感 2 个方面，使画中情感融人诗 中且能更好地从用户这一作诗者的角度出发得到 带有鲜明情感特征的诗句.

\section{参考文献(References):}

[1] Sutskever I, Vinyals O, Le Q V, et al. Sequence to sequence learning with neural networks[C] //Proceedings of the 27th International Conference on Neural Information Processing Systems. Cambridge: MIT Press, 2014: 3104-3112

[2] Krizhevsky A, Sutskever I, Hinton G E, et al. ImageNet classification with deep convolutional neural networks[C] //Proceedings of the 25th International Conference on Neural Information Processing Systems. Cambridge: MIT Press, 2012: 1097-1105

[3] Kulkarni G, Premraj V, Ordonez V, et al. BabyTalk: understanding and generating simple image descriptions[C] //Proceedings of the IEEE Conference on Computer Vision and Pattern Recognition. Los Alamitos: IEEE Computer Society Press, 2011: 1601-1608

[4] Farhadi A, Hejrati M, Sadeghi M A, et al. Every picture tells a story: generating sentences from images[C] //Proceedings of the 11th European Conference on Computer Vision. Heidelberg: Springer, 2010: 15-29

[5] Vinyals O, Toshev A, Bengio S, et al. Show and tell: lessons learned from the 2015 MSCOCO image captioning challenge[J]. IEEE Transactions on Pattern Analysis and Machine Intelligence, 2017, 39(4): 652-663

[6] Cho K, van Merrienboer B, Gulcehre C, et al. Learning phrase representations using RNN encoder-decoder for statistical machine translation[OL]. [2020-08-29]. http://arxiv.org/abs/1406. $1078 \mathrm{v} 3$

[7] Vinyals O, Toshev A, Bengio S, et al. Show and tell: a neural image caption generator[C] //Proceedings of the IEEE Conference on Computer Vision and Pattern Recognition. Los Alamitos: IEEE Computer Society Press, 2015: 3156-3164

[8] Karpathy A, Li F F. Deep visual-semantic alignments for generating image descriptions[J]. IEEE Transactions on Pattern Analysis and Machine Intelligence, 2017, 39(4): 664-676

[9] Szegedy C, Liu W, Jia Y Q, et al. Going deeper with convolutions[C] //Proceedings of the IEEE Conference on Computer Vision and Pattern Recognition. Los Alamitos: IEEE Computer Society Press, 2015: 1-9

[10] Simonyan K, Zisserman A. Very deep convolutional networks for large-scale image recognition[OL]. [2020-08-29]. http:// arxiv.org/abs/1409.1556v6

[11] Hochreiter S, Schmidhuber J. Long short-term memory[J]. Neural Computation, 1997, 9(8): 1735-1780

[12] Jia X, Gavves E, Fernando B, et al. Guiding the long-short term memory model for image caption generation[C] //Proce- edings of the IEEE International Conference on Computer Vision. Los Alamitos: IEEE Computer Society Press, 2015: 2407-2415

[13] Wu Q, Shen C H, Liu L Q, et al. What value do explicit high level concepts have in vision to language problems?[C] // Proceedings of the IEEE Conference on Computer Vision and Pattern Recognition. Los Alamitos: IEEE Computer Society Press, 2016: 203-212

[14] Mao J H, Xu W, Yang Y, et al. Explain images with multimodal recurrent neural networks[OL]. [2020-08-29]. http://arxiv.org/ abs/1410.1090v1

[15] Ren S Q, He K M, Girshick R, et al. Faster R-CNN: towards real-time object detection with region proposal networks[J]. IEEE Transactions on Pattern Analysis and Machine Intelligence, 2015, 39(6): 1137-1149

[16] He K M, Gkioxari G, Dollár P, et al. Mask R-CNN[J]. IEEE Transactions on Pattern Analysis and Machine Intelligence, 2020, 42(2): 386-397

[17] Pang J M, Chen K, Shi J P, et al. Libra R-CNN: towards balanced learning for object detection[C] //Proceedings of the IEEE Conference on Computer Vision and Pattern Recognition. Los Alamitos: IEEE Computer Society Press, 2019: 821-830

[18] Zhou Changle, You Wei, Ding Xiaojun. Genetic algorithm and its implementation of automatic generation of Chinese SONGCI [J]. Journal of Software, 2010, 21(3): 427-437(in Chinese) (周昌乐, 游维, 丁晓君. 一种宋词自动生成的遗传算法及 其机器实现[J]. 软件学报, 2010, 21(3): 427-437)

[19] He J, Zhou M, Jiang L. Generating Chinese classical poems with statistical machine translation models[C] //Proceedings of the 26th AAAI Conference on Artificial Intelligence. Palo Alto: AAAI Press, 2012: 1650-1656

[20] Zhang X X, Lapata M. Chinese poetry generation with recurrent neural networks[C] //Proceedings of the Conference on Empirical Methods in Natural Language Processing. Doha: EMNLP Press, 2014: 670-680

[21] Yan R. i, Poet: automatic poetry composition through recurrent neural networks with iterative polishing schema[C] //Proceedings of the 25th International Joint Conference on Artificial Intelligence. New York: IJCAI Press, 2016: 2238- 2244

[22] Yi X Y, Sun M S, Li R Y, et al. Chinese poetry generation with a working memory model[C] //Proceedings of the 27th International Joint Conference on Artificial Intelligence. Stockholm: IJCAI Press, 2018: 4553-4559

[23] Xing Shuo. Research on the model of generating Chinese poems from images based on neural network[D]. Hefei: Hefei University of Technology, 2018(in Chinese) (邢硕. 基于神经网络的看图写诗模型研究[D]. 合肥: 合肥 工业大学, 2018)

[24] Wang $\mathrm{Z}, \mathrm{He} \mathrm{W}, \mathrm{Wu} \mathrm{H}$, et al. Chinese poetry generation with planning based neural network[C] //Proceedings of COLING 2016. Osaka: COLING Press, 2016: 1051-1060

[25] Wang Dong, Bai Ziwei, Feng Yang, et al. The conversion method and device of modern prose to ancient poetry based on LSTM model: China, 201611140395.0[P]. 2017-04-26(in Chinese) (王东, 白紫薇, 冯洋, 等. 基于 LSTM 模型的现代文到古 诗的转换方法及装置: 中国, 201611140395.0[P]. 2017-0426)

[26] Szegedy C, Vanhoucke V, Ioffe S, et al. Rethinking the inception architecture for computer vision[C] //Proceedings of the IEEE Conference on Computer Vision and Pattern Recognition. Los Alamitos: IEEE Computer Society Press, 2016: 2818-2826 